\title{
Impact of Nap Length, Nap Timing and Sleep Quality on Sustaining Early Morning Performance
}

\author{
Tomohide KUBO ${ }^{1 *}$, Hidemaro TAKEYAMA ${ }^{1}$, Shun MATSUMOTO ${ }^{2}$, Takeshi EBARA ${ }^{1}$, \\ Kensaburo MURATA ${ }^{1}$, Norihide TACHI ${ }^{1}$ and Toru ITANI ${ }^{1}$ \\ ${ }^{1}$ Health Sciences of Life, Work and Environment, Nagoya City University Graduate School of Medical Sciences, \\ 1 Kawasumi, Mizuho-cho, Mizuho-ku, Nagoya 467-8601, Japan \\ ${ }^{2}$ The Institute of Science of Labor, 2-8-14 Sugao, Miyake-Ku, Kawasaki 216-8501, Japan
}

Received February 2, 2007 and accepted May 25, 2007

\begin{abstract}
The study examined how nap length, nap timing and sleep quality affect early morning performance (6:00 to 8:00). Twelve students participated in a simulated nightshift schedule (22:00 to 8:00) where the length and timing of nocturnal naps were manipulated $(0: 00-1: 00,0: 00-2: 00$, 4:00-5:00 and 4:00-6:00). A performance test battery was administered consisting of a psychomotor vigilance test, a logical reasoning test, and a visual analogue scale for subjective fatigue and sleepiness. The results showed that a 120-min nap sustained early morning performance better than a 60-min nap. Taking a nap earlier or later did not affect the neurobehavioral performance tests, although participants slept more efficiently during naps later in the night shift. A negative effect of a nocturnal nap during the night shift on subsequent daytime and nocturnal sleep was not observed in the sleep architecture. It still remains unclear whether slow wave sleep plays an important role in sustaining early morning performance. In terms of work safety and sleep health, the results suggest that a longer and later nap is beneficial during night shifts.
\end{abstract}

Key words: Countermeasure against night work, Fatigue, Nocturnal nap, Polysomnography, Shift work, Sleepiness, Sleep inertia

\section{Introduction}

In everyday life, people feel fatigue, which is a sign of needing a rest ${ }^{1,2}$. According to Bartley ${ }^{1}$, fatigue is a protective phenomenon that helps maintain the equilibrium of the organism by stimulating the desire for taking a rest. Therefore, management of fatigue is considered an important factor to sustain alertness and performance ${ }^{3,4)}$, reduce human error $^{5,6}$, reduce the incidence of diseases ${ }^{7-9}$, and increase the quality of life ${ }^{10,11)}$. Shift workers, however, often cannot take a rest in spite of noticing fatigue. Shift workers are compelled to work and sleep at irregular hours not consistent with their innate circadian rhythms, and thereby suffer from severe sleepiness and fatigue compared with day workers ${ }^{12,13)}$. Some researchers in Japan have investigated the effects of nocturnal

*To whom correspondence should be addressed. napping during duty as a nightshift countermeasure for the problem of sleepiness and fatigue in nightshift workers ${ }^{14-26)}$. According to these studies, nocturnal napping could reduce sleepiness and fatigue, and help maintain circadian rhythms, and thereby have beneficial effects for work safety, workers' sleep health and quality of life ${ }^{26}$. In other countries, there has been growing interested in nocturnal napping, especially for airline pilots ${ }^{27}$, physicians $^{28)}$, nurses ${ }^{28,29)}$, medical residents and interns ${ }^{30)}$, industrial plant operators ${ }^{31)}$, and aircraft maintenance engineers ${ }^{32}$.

However, the most effective type of nocturnal nap has not yet been determined ${ }^{33)}$. Only a few studies have investigated the timing and length of nocturnal naps. Sallinen et al. ${ }^{34)}$ investigated the efficiency of 30-min and 50-min nocturnal naps taken either in the earlier part of the nightshift (around 1:00) or in the latter part (around 4:00). They concluded that alertness could be improved by a nap of either 
length at either of those nap timings. In contrast, Gillberg ${ }^{35)}$ reported that a one-hour nap taken at 4:30 improved alertness during the early morning more than a one-hour nap taken at 21:00. While Bonnet ${ }^{36)}$ suggested that the effects of taking a nap on alertness and performance depended on the length of the nap, there are some findings that a short nap is effective for sustaining performance and alertness ${ }^{27,28,32,34)}$.

Although it has been reported that taking a nocturnal nap sustains early morning performance, little is known about what qualities of a nap are more beneficial. According to some studies, slow wave sleep (SWS) might have a restorative role for neurobehavioral functions ${ }^{37-40}$. SWS is a stage of deep sleep characterized by high-amplitude delta EEG waves $^{41)}$. Jurado et al. ${ }^{37)}$ observed a significant relationship between the amount of SWS and the improvement of reaction times. Recently, Walsh et al ${ }^{40)}$ showed that pharmacologic SWS enhancement improved neurobehavioral functions during four nights of sleep restriction. In contrast, Gillberg \& Åkerstedt ${ }^{42)}$ suggested that total sleep time might be more important for neurobehavioral performance than the amount of SWS. Therefore, studies investigating the relationship between SWS and neurobehavioral functions have produced inconsistent findings ${ }^{42-44)}$.

The purpose of the present study was to test the following three hypotheses.

Hypothesis 1: A 120-min nap will better sustain early morning performance than a 60-min nap will.

Hypothesis 2: A later nap will better sustain early morning performance than an earlier nap will.

Hypothesis 3: The amount of SWS during a nocturnal nap will have a positive relationship to better sustain early morning performance.

\section{Methods}

\section{Participants}

Participants were recruited from university students according to the following criteria: healthy male, "neither entirely morning nor evening type" evaluated by morningevening questionnaires ${ }^{45}$, non-smoker, and did not have a sleep disorder as assessed by a sleep disorder questionnaire and interview. Twelve male university students (Mean \pm SD; $21.6 \pm 2.8 \mathrm{yr}$ old) participated in this experiment. Participants gave informed consent in writing prior to the experiment. Ethical approval for this experiment was obtained from the Ethics Committee of Nagoya City University. All participants were paid for participation (150,000 yen).

\section{Design}

The experimental schedule is shown in Fig. 1. The experiment was composed of 5 conditions, each lasting 3 consecutive days with one night shift (22:00-8:00), followed by daytime sleep (11:30-17:30) and nocturnal sleep (0:007:00). The 5 different conditions manipulated the timing and length of naps: 0:00-1:00 (Early 60-min; E60), 0:002:00 (Early 120-min; E120), 4:00-5:00 (Late 60-min; L60), 4:00-6:00 (Late 120-min; L120) and no nap (Control). Participants completed all the experimental conditions in a counterbalanced order. To minimize carry-over effects, at least five days elapsed between each experimental condition.

\section{Measurements}

The performance test battery consisted of a modified version of psychomotor vigilance test (PVT), a logical reasoning test (LRT) and a visual analogue scale (VAS).

\section{1) Psychomotor Vigilance Test}

The PVT was used to evaluate sustained attention. Previous research reported that the PVT is highly sensitive to the effect of sleep loss and is minimally subject to learning effects $^{46,47)}$. The modified version of the PVT employed in this study required the participants to press the key corresponding to the numeral $(2,4,6,8)$ presented in the center of the display as quickly and accurately as possible. The stimulus was presented for $5 \mathrm{~s}$ and the inter-stimulus interval randomly varied between 1 and $10 \mathrm{~s}$. No response or a response later than $5 \mathrm{~s}$ was scored as a lapse in attention.

\section{2) Logical Reasoning Test}

The LRT developed by Baddeley ${ }^{48)}$ was used to evaluate working memory. Participants are randomly shown stimuli like "AB" or "BA", with logical statements such as "A is ahead of B", "A is not preceded by B", and so on. Participants were required to answer "True" or "False" by pressing the appropriate key as quickly and accurately as possible. In the LRT, both RTs and correct responses were recorded.

\section{3) Visual Analogue Scale}

The VAS was used to evaluate subjective sleepiness and fatigue. Participants rated their sleepiness and fatigue on a 100-mm line. The value ranged from $0 \mathrm{~mm}$ (not at all sleepy or tired) to $100 \mathrm{~mm}$ (extremely sleepy or tired).

\section{4) Polysomnography}

Electroencephalography (EEG), surface-electrode electromyography and bilateral electrooculography were recorded using silver-silver chloride electrodes during the 


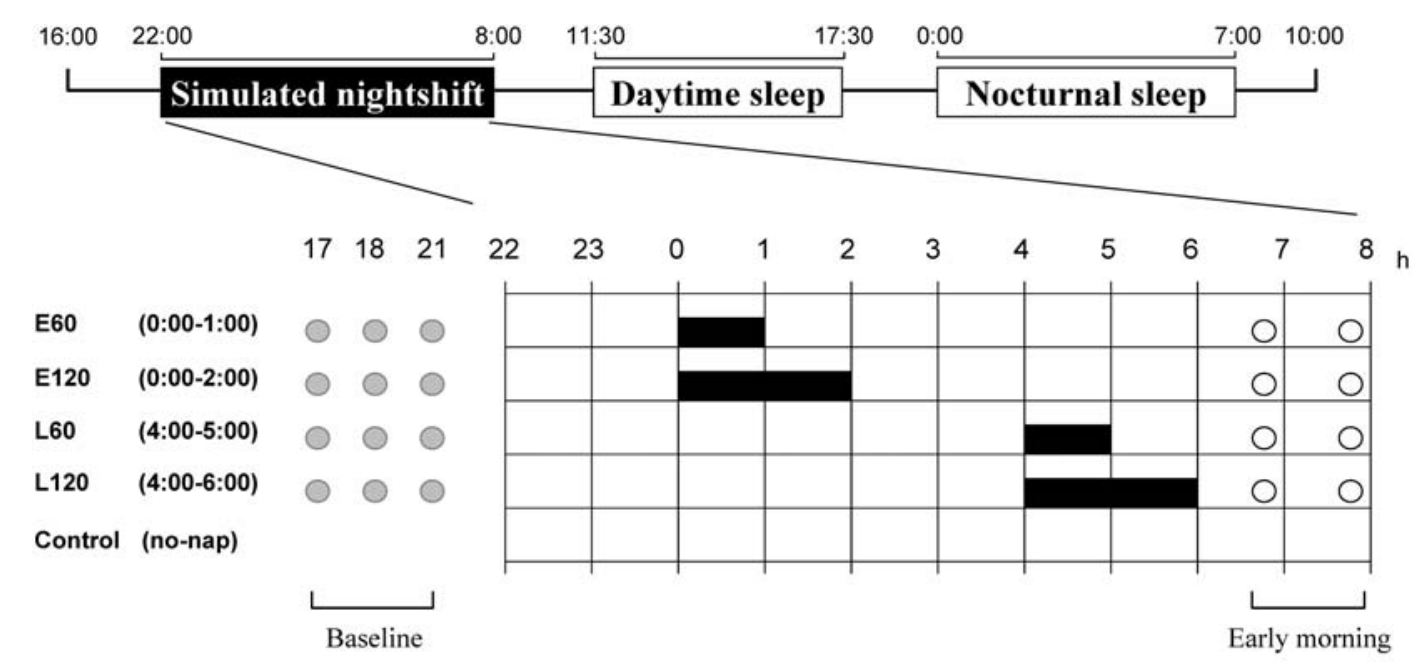

\section{Performance tests}

Fig. 1. Experimental Schedule.

nap periods on a Polymate AP1124 (TEAC Instruments Co., Ltd, Tokyo, Japan). The EEG was obtained from C4-A1 and C3-A2 derivation. Sleep stage 3 and 4 is a stage of deep sleep characterized by high-amplitude delta EEG waves (more than $75 \mathrm{mV}$, less than $2 \mathrm{~Hz}$ ) occupying $20-50 \%$ or more than $50 \%$ of the scoring epoch, respectively. Their sleep stages are combined to provide an estimate of slow wave sleep. The impedances were kept below 20,000 ohms. The $20 \mathrm{~s}$ EEG epochs were visually scored according to the standard criteria ${ }^{41}$. Scoring was facilitated by the sleep scoring software "Night Owl Stager ver.1.50A" (Norupro Light Systems Co., Ltd, Tokyo, Japan).

\section{Procedure}

Prior to the beginning of a series of experiments, participants spent one night in the laboratory to adapt to the laboratory environment. In addition, participants were trained on a performance test battery in order to familiarize them with the test battery and to minimize improvements in performance resulting from learning. During the training period, participants were required to repeat the test battery until their performance reached a plateau. The bedroom environment was kept quiet and free from interruptions. External time cues were eliminated during the experiments. Participants were instructed to do their usual daily activities and sleep $(0: 00-7: 00)$ before the experiments. On the day preceding the experiments, participants were prohibited from taking a daytime nap, or drinking beverages containing caffeine or alcohol. Participant's sleep-wake cycles were measured by an actigraph (A.M.I Co.; Micro-mini RR) and a self-reported sleep log. We confirmed that the participants followed the instructions.

Participants were required to arrive at the laboratory by 16:00 and practice a performance test battery. Participants ate dinner at 19:00, had a shower at 20:00, and were attached to the electrodes between 21:00 and 22:00. The simulated nightshift started at 22:00 and ended at 8:00. Participants were required to complete a work task $(30 \mathrm{~min})$, a performance test battery (20 min), and break (10 min) every hour during the simulated night shift. The work task was typing English documents into a computer. The test battery composed of three computer-administered performance tests. The work task and test battery were performed with the same computer.

Participants were served two biscuits and $60 \mathrm{ml}$ of mineral water every break time during the simulated nightshift to minimize sleepiness and irritability related to being hungry. Participants spent the break times reading a book and/or listening to music. Throughout the simulated nightshift, experimenters monitored the participants from a separate room. If participants fell asleep, they were awakened by an interphone alarm.

\section{Data analysis}

Before the statistical analyses, the distributions of all variables were checked. To reduce heterogeneity of variance between participants, RTs and lapses were transformed to their logarithms and square roots, respectively.

The effects of length and timing of a nocturnal nap on nap sleep physiology were analyzed with two-way repeated 
measures ANOVAs. Since there are some previous researches ${ }^{33,34,49)}$ suggesting that a nocturnal nap during night shift might disturb the following daytime sleep and nocturnal sleep, which might produce negative influences on alertness during the next night shift, the possible effects of the naps on physiological variables associated with daytime sleep and nocturnal sleep were analyzed with one-way repeated measures ANOVAs. Post-hoc analyses were performed using Tukey HSD tests to specify differences among the mean values.

For the neurobehavioral performance and subjective alertness data (i.e. PVT, LRT and VAS), the average of the values at $17 \mathrm{~h}(17: 30-17: 50), 18 \mathrm{~h}(18: 30-18: 50)$ and $21 \mathrm{~h}$ (21:30-21:50) was used as the baseline session value and the averages at $6 \mathrm{~h}(6: 30-6: 50)$ and $7 \mathrm{~h}(7: 30-7: 50)$ was used as the value for the early morning session. The differences between baseline and early morning values were analyzed to examine the impacts of nap length and nap timing on sustaining early morning performance. The impacts of length and timing of a nocturnal nap on early morning performance were analyzed with two-way repeated measures ANOVAs for "nap length" (60 min vs. $120 \mathrm{~min}$ ) and "nap timing" (early vs. late). To investigate whether SWS and total sleep time contributed to the effects of nocturnal naps, Pearson's product moment correlation coefficients were calculated between the sleep variables and the neurobehavioral performance and subjective alertness variables for each nap condition.

The Huynh \& Feldt's epsilon correction was employed when appropriate, but the original degrees of freedom are reported in this paper. The significance level was set at $p=0.05$. All statistical analyses were performed using SPSS 14.0J for Windows.

\section{Results}

\section{Polysomnographic variables}

1) Nocturnal nap during simulated night shift

The results for the polysomnographic variables during nocturnal naps are presented in Table 1 . There was a significant main effect of Nap Timing for Sleep Efficiency $\left(\mathrm{F}_{1,11}=6.88, p=0.023\right)$. The effect of Nap Length was significant for Total Sleep Time $\left(\mathrm{F}_{1,11}=396.08, p<0.001\right)$, Stage $2\left(\mathrm{~F}_{1,11}=232.65, p<0.001\right)$, Stage $3\left(\mathrm{~F}_{1,11}=7.59, p<\right.$ $0.019)$, Stage $4\left(\mathrm{~F}_{1,11}=33.41, p<0.001\right)$ and Stage SWS $\left(\mathrm{F}_{1,11}=35.48, p<0.001\right)$. The interaction of Nap Timing and Nap Length was not significant.

2) Daytime sleep after the naps

For sleep obtained between 11.30 and 17.30, significant effects of the nocturnal naps on daytime sleep architecture were found for Stage 3, Stage 4, SWS and REM (Table 1). The amount of SWS in the control condition was greater than in the other conditions (i.e., E60, E120, L60, and L120). In contrast, the time spent in REM was shorter in the control condition than in the other conditions. For the other polysomnographic variables, there were no significant differences between conditions.

3) Nocturnal sleep after the daytime sleep

For the polysomnographic variables during nocturnal sleep (0:00-7:00), there were no significant findings (Table 1). Possible effects of the naps on physiological variables associated with nocturnal sleep were not found.

\section{Early morning performance}

Table 2 summarizes ANOVA results from visual analogue scale, psychomotor vigilance test and logical reasoning test.

1) Visual analogue scale

The VAS indicates the effect of nocturnal naps on subjective sleepiness and fatigue. Figure 2 shows the change in the subjective alertness variables from the baseline session to the early morning session. A significant main effect of Nap Timing was observed on the VAS for fatigue $\left(\mathrm{F}_{1,11}=12.949, p=0.004\right)$. Regarding sleepiness, there were no significant findings on the VAS. For both VAS ratings, the interaction of Nap Timing and Nap Length was not significant.

\section{2) Psychomotor vigilance test}

The PVT indicates the effect of nap length and nap timing on sustained attention. Figure 3 shows the change in the PVT variables from the baseline session to the early morning session. ANOVAs showed that there were significant main effects of Nap Length for the slowest $10 \%$ of the reaction times $\left(\mathrm{F}_{1,11}=5.302, p=0.042\right)$ and lapses $\left(\mathrm{F}_{1,11}=5.451\right.$, $p=0.040$ ). The interaction of Nap Timing and Nap Length was not significant for the PVT for the slowest $10 \%$ of the reaction times and lapses.

\section{3) Logical reasoning test}

The LRT indicates the effect of nap length and nap timing on working memory. Figure 4 shows the change in the LRT variables from the baseline session to the early morning session. There were no significant findings for the LRT variables. 


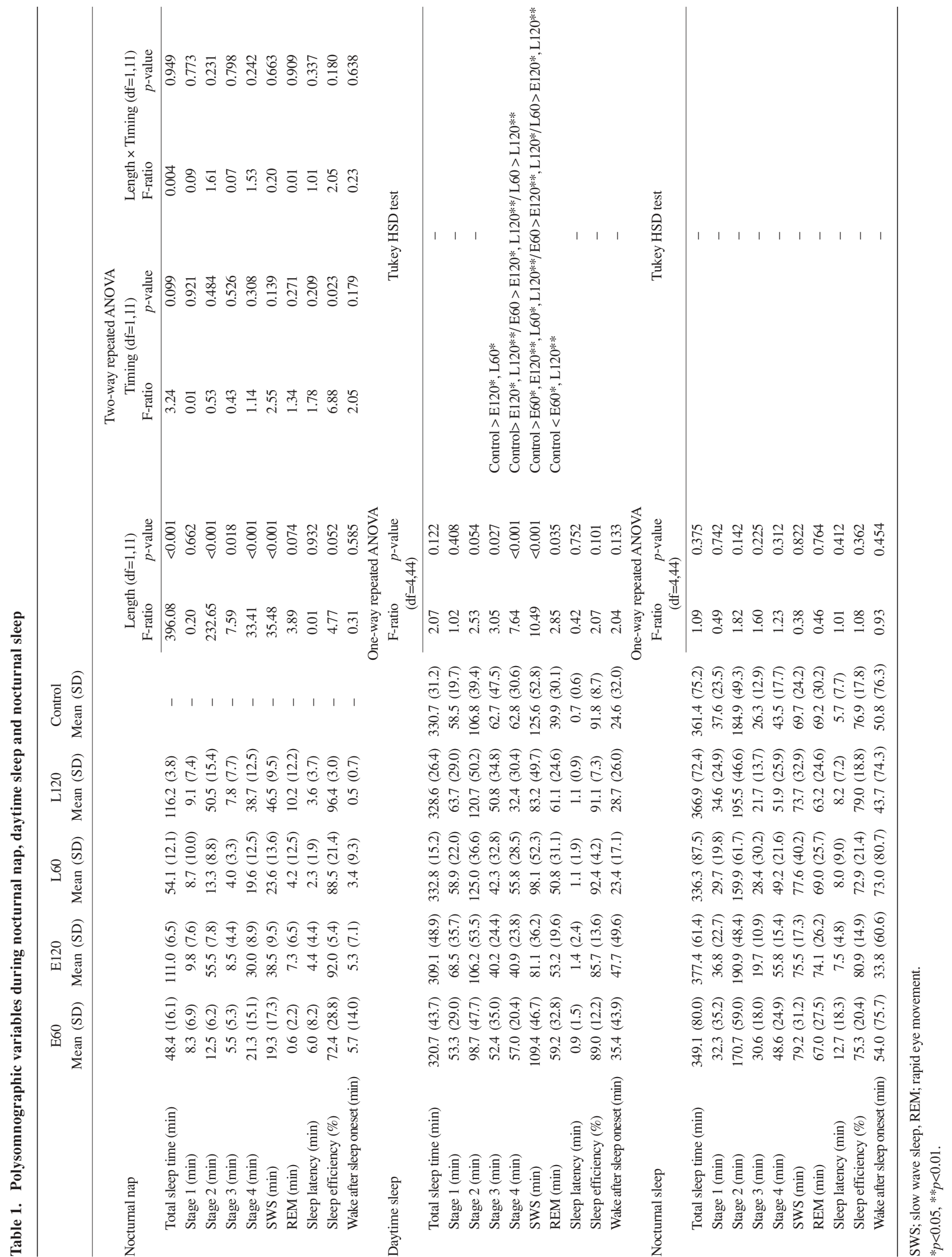


Table 2. Summary of ANOVA results from visual analogue scale, psychomotor vigilance test and logical reasoning test

\begin{tabular}{|c|c|c|c|c|c|c|c|}
\hline \multirow{3}{*}{ Parameter } & & \multicolumn{6}{|c|}{ Two-way repeated measure ANOVA } \\
\hline & & F-ratio & $p$-value & F-ratio & $p$-value & F-ratio & $p$-value \\
\hline & & \multicolumn{2}{|c|}{ Length $(\mathrm{df}=1,11)$} & \multicolumn{2}{|c|}{ Timing $(\mathrm{df}=1,11)$} & \multicolumn{2}{|c|}{ Length $\times$ Timing $(\mathrm{df}=1,11)$} \\
\hline \multicolumn{8}{|c|}{ Visual Analogue Scale } \\
\hline & Sleepiness & 0.041 & 0.843 & 0.291 & 0.600 & 0.106 & 0.751 \\
\hline & Fatigue & 1.378 & 0.265 & 12.949 & $0.004 * *$ & 0.976 & 0.344 \\
\hline \multicolumn{8}{|c|}{ Psychomotor Vigilance Test } \\
\hline & Slowest $10 \%$ Reaction time & 5.302 & $0.042 *$ & 0.755 & 0.403 & 0.170 & 0.688 \\
\hline & Lapse & 5.451 & $0.040 *$ & 0.689 & 0.737 & 0.037 & 0.850 \\
\hline \multicolumn{8}{|c|}{ Logical Reasoning Test } \\
\hline & Reaction time & 0.119 & 0.737 & 0.013 & 0.911 & 0.922 & 0.358 \\
\hline & Correct response & 2.433 & 0.147 & 0.232 & 0.640 & 0.387 & 0.547 \\
\hline
\end{tabular}

$* p<0.05, * * p<0.01$.

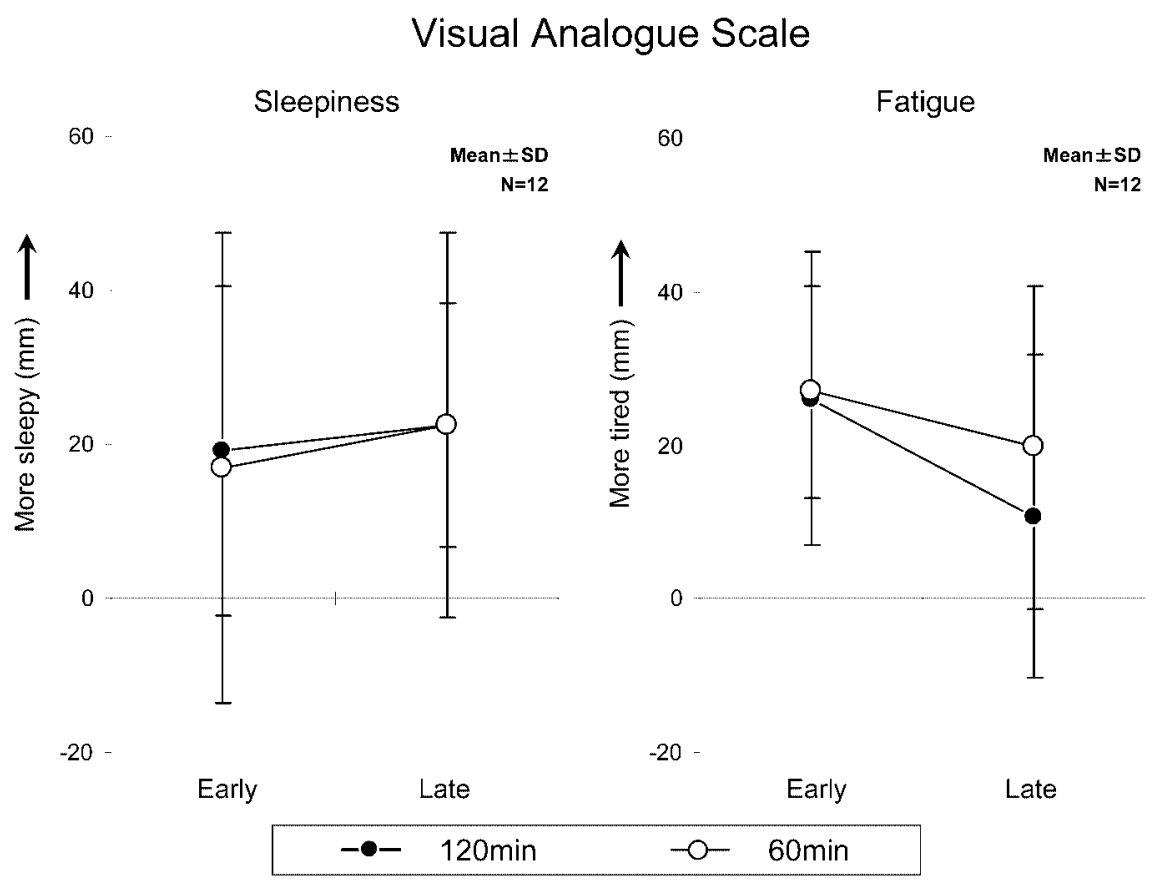

Fig. 2. The effect of nap length and nap timing on subjective alertness during early morning. The data are expressed as the mean (SD) of the changes in the early morning performance compared to the baseline.

Polysomnographic variables and early morning performance

To investigate the relationship between the polysomnographic variables and the early morning performance data, correlation coefficients were calculated (Table 3). For the L60 condition, the amount of SWS was significantly correlated with the VAS ratings for sleepiness $(\mathrm{r}=-0.64, p<0.01)$ and fatigue $(\mathrm{r}=-0.76, p<0.01)$. In addition, moderate non-significant correlations were observed between the amount of SWS and the LRT for correct responses in the $\mathrm{L} 60$ condition $(\mathrm{r}=0.42)$, and reaction times in the E60 condition $(\mathrm{r}=-0.40)$. No significant correlations were observed for the amount of total sleep time. However, the VAS for sleepiness $(r=0.42)$ and the PVT for slowest reaction times ( $\mathrm{r}=0.43)$ and lapses $(\mathrm{r}=0.49)$ in the $\mathrm{L} 120$ moderately correlated with the amount of total sleep time. 


\section{Psychomotor Vigilance Test}

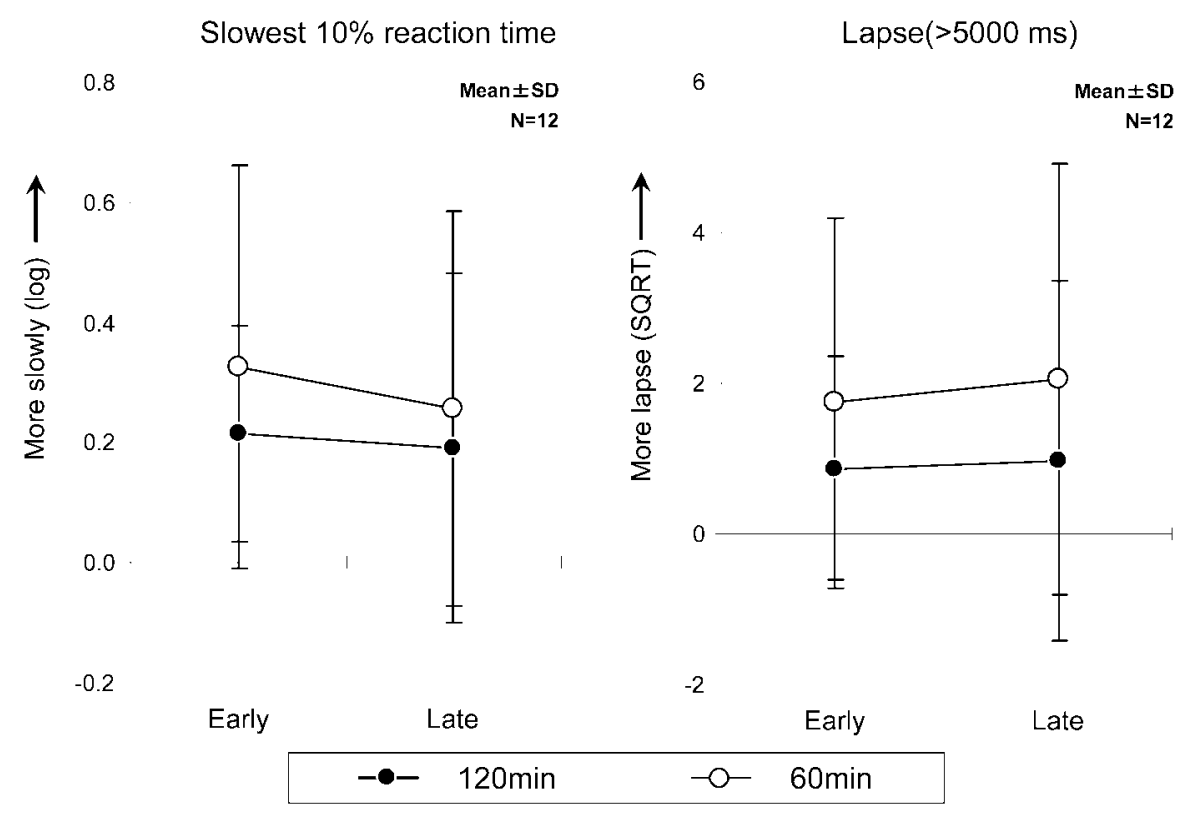

Fig. 3. The effect of nap length and nap timing on sustained attention during early morning. The data are expressed as the mean (SD) of the changes in the early morning performance compared to the baseline.

\section{Discussion}

The purpose of the present study was to test three hypotheses concerning the impact of nap length, nap timing and sleep quality on sustaining early morning performance.

\section{Hypothesis 1: Nap length and early morning performance}

The polysomnographic variables (i.e., Total sleep time, Stage 2, 3, 4 and SWS) for the 120-min nap conditions were significantly increased compared to the 60-min nap conditions (Table 1). For the PVT performance, the significant improvements for the 120-min nap conditions were observed compared to the 60-min nap conditions (Fig. $3)$. These results are consistent with the study of Bonnet ${ }^{36)}$. They examined the effect of naps $(2,4$ or $8 \mathrm{~h})$ placed prior to two consecutive night of sleep deprivation. As a result, performance and alertness in all nap conditions were improved in a dose-response fashion compared to the nonap condition. Therefore, they concluded that the extent of improvement is dependent on the nap length.

However, the results for the LRT and VAS in this study did not reveal whether taking a 60-min nap or a 120 -min nap is more beneficial during the night shift. The factors behind the discrepancy among these results might be related to the measurements' sensitivity for sleep loss. Other studies showed that a simple monotonous task (like the PVT) is highly sensitive to the effect of sleep loss, while a complex task (like the LRT) is not sensitive ${ }^{50)}$. Another possibility is that the results on the tests are influenced by the circadian rhythm, since the evaluation time (6-7 h) was early morning. It is possible that the participants' performance deterioration at the evaluation time might have been alleviated by innate circadian rhythms regardless of taking naps. Therefore, one night shift might not be sufficient to show the effect of naps on neurobehavioral performance and subjective alertness. Further investigations with consecutive night shifts may be necessary to show more clearly the effect of naps.

The problem we have to consider next is the effect of a nocturnal nap on the following daytime and nocturnal sleep from the viewpoint of workers' sleep health. Opinions are divided between researchers in Japan and other countries. Researchers in other countries considered that a nocturnal nap during night shift might disturb the following daytime sleep, which might produce negative influences on alertness during the next night shift ${ }^{33,34,49)}$. In contrast, some researchers in Japan ${ }^{18,24-26)}$ suggested that the effect of a nocturnal nap on subsequent daytime sleep might be interpreted as beneficial because workers can spend private time engaged in other activities instead of sleeping. 


\section{Logical Reasoning Test}

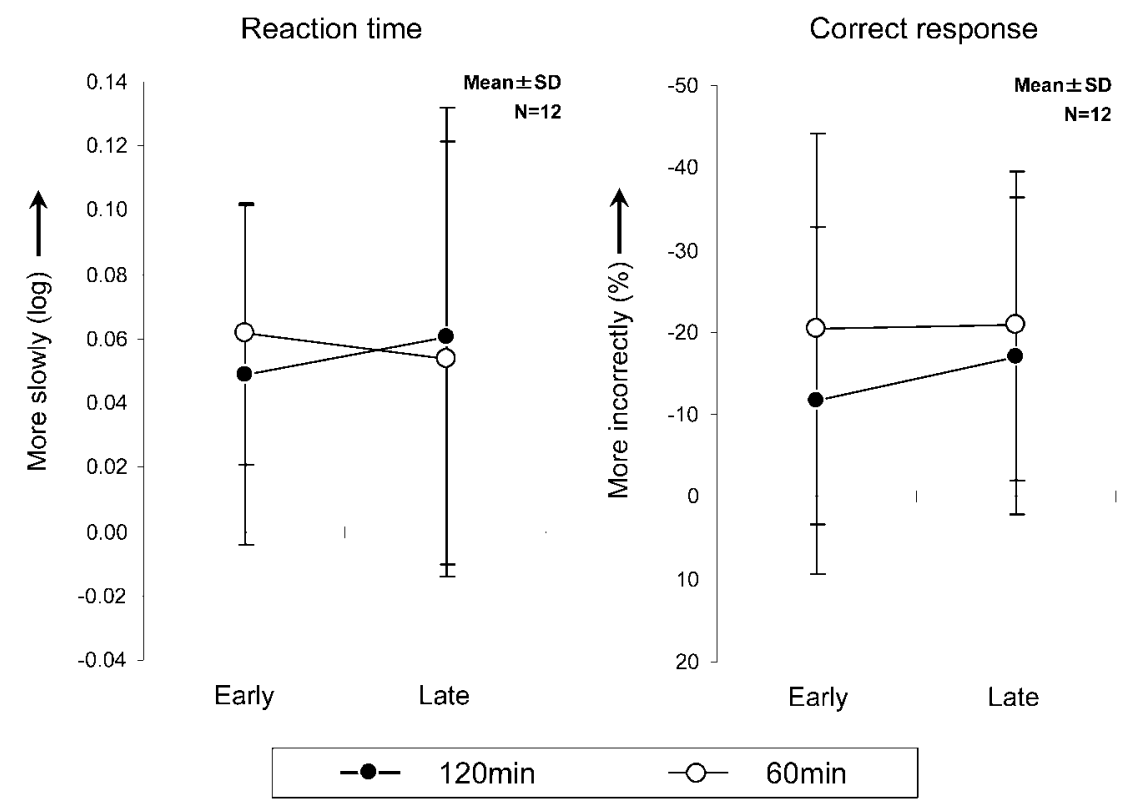

Fig. 4. The effect of nap length and nap timing on working memory during early morning. The data are expressed as the mean (SD) of the changes in the early morning performance compared to the baseline.

Recently, Sasaki et al. ${ }^{25}$ investigated the effect of nocturnal naps taken during the simulated two consecutive night shifts on the sleep architecture of subsequent daytime and nighttime sleep. They showed that the nocturnal nap increased the wake time during the last part of the subsequent daytime sleep, and did not impair the sleep architecture of the subsequent nighttime sleep, compared to the baseline sleep before the night shift. Generally, for normal night sleep, the length of rapid eye movement (REM) sleep predominates compared to the length of SWS (i.e., length of stage REM sleep $>$ SWS ${ }^{51}$ ). However, due to a longer prior wakefulness time, in the sleep architecture of daytime sleep after a night shift, SWS predominates and the length of stage REM sleep is decreased, unlike the sleep architecture for a normal night sleep.

Our results showed that nocturnal naps decreased the SWS pressure and increase the duration of stage REM in daytime sleep, thereby making the participants' SWS-REM sleep balance ${ }^{25,52)}$ better in the sleep architecture of the subsequent daytime sleep (Table 1). This tendency was clearly observed in the longer nap conditions (i.e., E120 and L120). For the subsequent nocturnal sleep, no significant negative effects of nocturnal naps on the subsequent nocturnal sleep were observed in this study. On the basis of these results, we speculate that the effect of a nocturnal nap during night shift on the subsequent daytime sleep may not be negative.
Altogether, our results for the PVT performance support Hypothesis 1 that a 120-min nap would better sustain early morning performance than a 60-min nap. Therefore, in terms of work safety, we recommend a 120-min nap compared to a 60-min nap. Particularly, shift workers who require sustained attention (e.g., monitoring, driving, etc.) are encouraged to take a 120-min nap compared to a 60-min nap. Furthermore, in terms of sleep health, we recommend the longer nap to facilitate a suitable SWS-REM sleep balance, although additional research with more participants is needed to confirm this recommendation.

\section{Hypothesis 2: Nap timing and early morning performance}

The most effective nap timing remains to be determined in conjunction with the nap length. According to Matsumoto ${ }^{15}$, the most favorable nap timing to sustain performance and alertness during the night shift is around the nadir of body temperature. The finding is consistent with the research of Daurat $\&$ Foret ${ }^{29)}$. They reported that nurses working in an intensive care unit on a two 12-h shift system more frequently took a nap between 4:00 am and 6:00 am and felt that they needed to take a nap during the period. In contrast, Dinges et al. ${ }^{53)}$ recommended that a nap is most efficient if taken before sleep loss is felt (i.e., a "prophylactic nap" which is taken prior to night 
Table 3. The relationship between sleep quality and early morning performance

\begin{tabular}{|c|c|c|c|c|}
\hline Pearson product-moment correlation coefficient & E60 & E120 & L60 & L120 \\
\hline \multicolumn{5}{|l|}{ Slow wave sleep (min) } \\
\hline \multicolumn{5}{|l|}{ Visual Analogue Scale } \\
\hline Sleepiness & 0.31 & 0.02 & $-0.64 * *$ & 0.37 \\
\hline Fatigue & -0.24 & 0.01 & $-0.76^{* *}$ & -0.34 \\
\hline \multicolumn{5}{|l|}{ Psychomotor Vigilance Test } \\
\hline Slowest $10 \%$ Reaction time & 0.33 & 0.19 & -0.32 & 0.28 \\
\hline Lapse & 0.22 & 0.23 & -0.30 & 0.18 \\
\hline \multicolumn{5}{|l|}{ Logical Reasoning Test } \\
\hline Reaction time & -0.40 & 0.33 & -0.16 & 0.31 \\
\hline Correct response & -0.11 & 0.21 & 0.42 & -0.28 \\
\hline \multicolumn{5}{|l|}{ Total sleep time (min) } \\
\hline \multicolumn{5}{|l|}{ Visual Analogue Scale } \\
\hline Sleepiness & 0.18 & 0.32 & -0.11 & 0.42 \\
\hline Fatigue & 0.16 & 0.07 & -0.02 & 0.36 \\
\hline \multicolumn{5}{|l|}{ Psychomotor Vigilance Test } \\
\hline Slowest $10 \%$ Reaction time & 0.34 & 0.33 & 0.29 & 0.43 \\
\hline Lapse & 0.20 & 0.32 & 0.22 & 0.49 \\
\hline \multicolumn{5}{|l|}{ Logical Reasoning Test } \\
\hline Reaction time & -0.15 & -0.10 & 0.12 & -0.32 \\
\hline Correct response & -0.12 & -0.39 & -0.37 & -0.28 \\
\hline
\end{tabular}

shift). There is, however, relatively little research examining the effect of nap timing during night shift other than that of nap length.

The present study did not show a difference on the neurobehavioral performance tests in the effect of taking a nap earlier or later. One possible explanation is that the interval between the earlier and later naps in the present study is shorter (i.e., $2-3 \mathrm{~h}$ ) than in the previous study (i.e., $7.5 \mathrm{~h})^{35}$. It is possible that differences among the nap conditions were not found due to the short interval. When taking a nocturnal nap during the night shift, the effect of nap timing on early morning performance may not be more important than nap length.

However, it should be noted that subjective fatigue was rated significantly lower in the later nap conditions compared to the earlier nap conditions (Fig. 2). This may be related to the finding that participants slept more efficiently during the naps later in the night shift (Table 1). In contrast, there was no significant finding for subjective sleepiness in this study. This discrepancy may be related to the difference between sleepiness and fatigue. Fatigue is usually canceled by sleep or rest; while sleepiness does not necessary disappear immediately after sleep since it is increased by sleep inertia. According to previous studies $^{54}$, sleep inertia is a transitional state of lowered arousal occurring immediately after awakening from deep sleep. In the work place, performance deterioration due to sleep inertia is considered to be a potential problem of nocturnal napping ${ }^{33,54)}$. Some researchers suggested that the time course of sleep inertia can last from 1 min to 4 $\mathrm{h}^{54)}$. So the effect of sleep inertia might have contributed to the results for subjective sleepiness. If this is the case, the VAS ratings for sleepiness in the later nap conditions could be improved by a strategy for reducing sleep inertia (e.g., caffeine, bright light, and face washing ${ }^{55}$ ). Therefore, we recommend a later nocturnal nap as a night shift countermeasure, if techniques combining nocturnal napping and a strategy for minimizing sleep inertia are used.

Taken together, the subjective fatigue data suggests that a later nap will better sustain performance in the early morning compared to an earlier nap. In contrast, for other variables, the data failed to support Hypothesis 2. We consider that nap timing is not a more important factor for sustaining early morning performance than nap length, if more than a 1-h nap is available in a workplace.

\section{Hypothesis 3: Sleep quality and early morning performance}

The results of previous studies examining the relationship between SWS and neurobehavioral performance have been contradictory and inconclusive, although previous research 
suggested that increased SWS facilitates ongoing cortical recovery from prior awake activities according ${ }^{38)}$. Unfortunately, in this study, a positive relationship between the amount of SWS and early morning performance could not be clearly observed. However, significant strong correlations between the amount of SWS and subjective alertness (sleepiness, $\mathrm{r}=-0.64, p<0.01$; fatigue, $\mathrm{r}=-0.76$, $p<0.01$ ) were found, especially for the L60 condition. Additionally, there was also a non-significant moderate correlation between the amount of SWS and the LRT for correct responses $(r=0.42)$. Therefore, the results for the L60 condition suggest that the amount of SWS during a nocturnal nap has a positive relationship to better sustaining early morning performance. In contrast, there were no significant findings in the other conditions.

Based on these results, several possibilities remain to be tested in further investigations. One is that the relationship between the amount of SWS and early morning performance may be a U-shape function, not a linear relationship. Paradoxically, the larger amount of SWS may contribute to a deterioration of early morning performance, although sustaining early morning performance would be directly related to the length of SWS during the nap. According to previous studies ${ }^{54}$, a larger amount of SWS contributed to sleep inertia and thereby performance deterioration. Thus, we speculate that an appropriate amount of SWS might exist, although our present data cannot address this.

Another possibility is that sleep factors other than SWS may be contributing to sustaining early morning performance. As shown in Table 3, our results are not consistent with the study of Gillberg and Åkerstedt ${ }^{42)}$ who suggested that total sleep time might be more important for performance and alertness than the amount of SWS. On the other hand, Stickgold et al. ${ }^{56)}$ suggested that REM sleep plays an important role in memory consolidation. However, a limitation of our study is that we could not analyze the effect of REM sleep on early morning performance because the sample of REM sleep was too small for a statistical analysis. Further testing is needed with larger samples.

Taken altogether, it is still unclear whether the amount of SWS during naps is related to sustaining early morning performance. Therefore, we failed to support Hypothesis 3 that the amount of SWS during a nocturnal nap will have a positive relationship to sustaining early morning performance. However, we suggest that, for a nap shorter than $1 \mathrm{~h}$, the amount of SWS may be related to sustaining early morning performance in a linear function, although additional research would be needed to confirm this.

\section{Conclusion}

In work places where a nocturnal nap during duty can be arranged with comparative ease, we recommend longer and later napping to sustain early morning performance in terms of work safety and sleep health. However, if only a short nap can be arranged in a workplace, we consider that an earlier nap might be better than a later nap because of the greater effect of sleep inertia on morning performance from a later nap compared to an earlier nap. Therefore, especially for later naps, we suggest that techniques combining nocturnal napping and a strategy for sleep inertia are more effective. For sleep quality, it still remains unclear whether SWS plays a role in sustaining early morning performance.

Since the participants in the current study took naps in an environment where noise, light and social contact were controlled, it is possible that participants' sleep and performance may be better than real shift workers. Especially in real situations, daytime sleep after a night shift is often disturbed by these factors. Therefore, further investigation in real workplaces is necessary.

\section{Acknowledgement}

The study was supported by a Grant-in-Aid for Scientific Research (B) (2) 14370142 from the Ministry of Education, Culture, Sports, Science and Technology, Japan.

\section{References}

1) Bartley SH (1965) The management of fatigue. In: Fatigue: Mechanism and management, Kugelmass IN (Ed.), 72-86, Charles C Thomas Pub Ltd, Springfield, Illinois.

2) Saito Y (1995) The concept of over-fatigue essential for industrial fatigue research. J Sci Labour 71, 1-9 (in Japanese with English abstract).

3) Belenky G, Wesensten NJ, Thorne DR, Thomas ML, Sing HC, Redmond DP, Russo MB, Balkin TJ (2003) Patterns of performance degradation and restoration during sleep restriction and subsequent recovery: a sleep dose-response study. J Sleep Res 12, 1-12.

4) Van Dongen HP, Maislin G, Mullington JM, Dinges DF (2003) The cumulative cost of additional wakefulness: dose-response effects on neurobehavioral functions and sleep physiology from chronic sleep restriction and total sleep deprivation. Sleep 15, 117-26.

5) Bonnet MH, Arand DL (1995) We are chronically sleep deprived. Sleep 18, 908-11.

6) Folkard S, Tucker P (2003) Shift work, safety and productivity. Occup Med 53, 95-101.

7) Åkerstedt T, Fredlund P, Gillberg M, Jansson B (2002) Work 
load and work hours in relation to disturbed sleep and fatigue in a large representative sample. J Psychosom Res 53, 585-8.

8) Meier-Ewert HK, Ridker PM, Rifai N, Regan MM, Price NJ, Dinges DF, Mullington JM (1999) Effect of sleep loss on C-reactive protein, an inflammatory marker of cardiovascular risk. J Am Coll Cardiol 18, 678-83.

9) Spiegel K, Leproult R, Van Cauter E (1999) Impact of sleep debt on metabolic and endocrine function. Lancet 23, 1435-9.

10) Costa G (1996) The impact of shift and night work on health. Appl Ergon 27, 9-16.

11) Åkerstedt $T$, Olsson $B$, Ingre $M$, Holmgren $M$, Kecklund $G$ (2001) A 6-hour working day-effects on health and wellbeing. J Hum Ergol 30, 197-202.

12) Kogi K (1982) Sleep problems in night and shift work. J Hum Ergol 11 (Suppl), 217-31.

13) Rutenfranz J (1982) Occupational health measures for night and shift workers. J Hum Ergol 11 (Suppl), 67-86.

14) Kogi K (1962) On the present status of shift formation in Japan and mining. J Sci Lab 38, 135-45.

15) Matsumoto K (1981) Effects of nighttime naps on body temperature changes, sleep patterns, and self-evaluation of sleep. J Hum Ergol 10, 173-84.

16) Sakai K, Kogi K (1986) Conditions for three-shift workers to take nighttime naps effectively. In: Night and shift work: Long term effects and their prevention, Haider M, Koller M, Cervinka R (Eds.), 173-80, Verlag Peter Lang, Frankfurt am Main.

17) Matsumoto K, Morita $Y$ (1987) Effects of nighttime nap and age on sleep patterns of shift workers. Sleep 10, 580-9.

18) Matsumoto K, Harada M (1994) The effect of night-time naps on recovery from fatigue following night work. Ergonomics 37, 899-907.

19) Saito Y, Sasaki T (1996) The effect of length of a nocturnal nap on fatigue feelings during subsequent early morning hours. J Sci Labour 72, 15-23.

20) Takahashi M, Arito H, Fukuda H (1999) Nurses' workload associated with 16-h night shifts. II: Effects of a nap taken during the shifts. Psychiatry Clin Neurosci 53, 223-5.

21) Kubo T, Sasaki $T$ (2000) As a night shift countermeasure during early morning hours, which is more preferable, prophylactic napping or maintenance napping?-An experimental study. J Sci Labour 76, 473-86 (in Japanese with English abstract).

22) Takeyama H, Matsumoto S, Murata K, Ebara T, Kubo T, Tachi N, Itani T (2004) Effects of the length and timing of nighttime naps on task performance and physiological function. Rev Saude Publica 38 (Suppl), 32-7.

23) Takeyama H, Itani T, Tachi N, Sakamura O, Murata K, Inoue T, Takanishi T, Suzumura H, Niwa S (2005) Effects of shift schedules on fatigue and physiological functions among firefighters during night duty. Ergonomics 48, 1-11.

24) Takeyama H, Kubo T, Itani $T$ (2005) The nighttime nap strategies for improving night shift work in workplace. Ind Health 43, 24-9.

25) Sasaki T, Suzuki K, Kubo T, Matsumoto S, Matsukuma Y (2005)
Effects on sleep architecture of naps taken on the simulated two-consecutive night shifts. J Sci Labour 81, 161-8.

26) Sasaki T, Saito Y, Kikuchi Y (1992) A review of studies on the effects of nocturnal naps. J Sci Labour 68, 47-59 (in Japanese with English abstract).

27) Rosekind MR, Graeber RC, Dinges DF, Connell LJ, Rountree MS, Spinweber CL, Gillen KA (1994) Crew factors in flight operations IX, Effects of planned cockpit rest on crew performance and alertness in long-haul operations, Technical Memo 108839, NASA1994, Washington, DC.

28) Smith-Coggins R, Howard SK, Mac DT, Wang C, Kwan S, Rosekind MR, Sowb Y, Balise R, Levis J, Gaba DM (2006) Improving alertness and performance in emergency department physicians and nurses: the use of planned naps. Ann Emerg Med 48, 596-604.

29) Daurat A, Foret J (2004) Sleep strategies of 12-hour shift nurses with emphasis on night sleep episodes. Scand J Work Environ Health 30, 299-305.

30) Arora V, Dunphy C, Chang VY, Ahmad F, Humphrey HJ, Meltzer D (2006) The effects of on-duty napping on intern sleep time and fatigue. Ann Intern Med 144, 792-8.

31) Bonnefond A, Muzet A, Winter-Dill AS, Bailloeuil C, Bitouze F, Bonneau A (2001) Innovative working schedule: introducing one short nap during the night shift. Ergonomics 44, 937-45.

32) Purnell MT, Feyer AM, Herbison GP (2002) The impact of a nap opportunity during the night shift on the performance and alertness of 12-h shift workers. J Sleep Res 11, 219-27.

33) Bonnefond A, Tassi P, Roge J, Muzet A (2004) A critical review of techniques aiming at enhancing and sustaining worker's alertness during the night shift. Ind Health 42, 1-14.

34) Sallinen M, Härmä M, Åkerstedt T, Rosa R, Lillqvist O (1998) Promoting alertness with a short nap during a night shift. J Sleep Res 7, 240-7.

35) Gillberg M (1984) The effects of two alternative timings of a one-hour nap on early morning performance. Biol Psychol 19, 45-54.

36) Bonnet MH (1991) The effect of varying prophylactic naps on performance, alertness and mood throughout a 52-hour continuous operation. Sleep 14, 307-15.

37) Jurado JL, Luna-Villegas G, Buela-Casal G (1989) Normal human subjects with slow reaction times and larger time estimations after waking have diminished delta sleep. Electroencephalogr Clin Neurophysiol 73, 124-8.

38) Horne JA (1992) Human slow wave sleep: a review and appraisal of recent findings, with implications for sleep functions, and psychiatric illness. Experientia 15, 941-54.

39) Kecklund G, Åkerstedt T (2004) Apprehension of the subsequent working day is associated with a low amount of slow wave sleep. Biol Psychol 66, 169-76.

40) Walsh JK, Randazzo AC, Stone K, Eisenstein R, Feren SD, Kajy S, Dickey P, Roehrs T, Roth T, Schweitzer PK (2006) Tiagabine is associated with sustained attention during sleep restriction: evidence for the value of slow-wave sleep enhancement? Sleep 1, 433-43. 
41) Rechtschaffen A, Kales A (1968) A manual of standardized terminology, techniques and scoring system for sleep stages of human subjects, UCLA Brain Information Service/ Brain research Institute, Los Angeles, CA.

42) Gillberg M, Åkerstedt T (1994) Sleep restriction and SWSsuppression: effects on daytime alertness and night-time recovery. J Sleep Res 3, 144-51.

43) Edinger JD, Glenn DM, Bastian LA, Marsh GR (2000) Slowwave sleep and waking cognitive performance II: Findings among middle-aged adults with and without insomnia complaints. Physiol Behav 70, 127-34.

44) Spiegel R, Koberle S, Allen SR (1986) Significance of slow wave sleep: considerations from a clinical viewpoint. Sleep 9, 66-79.

45) Horne JA, Östberg O (1976) A self-assessment questionnaire to determine morningness - eveningness in human circadian rhythms. Int J Chronobiol 4, 97-110.

46) Dinges DF, Powell JW (1985) Microcomputer analyses of performance on a portable, simple visual RT task during sustained operations. Behav Res Methods Instrum Comput 17, 652-5.

47) Drummond SP, Bischoff-Grethe A, Dinges DF, Ayalon L, Mednick SC, Meloy MJ (2005) The neural basis of the psychomotor vigilance task. Sleep 28, 1059-68.

48) Baddeley AD (1968) A 3 min reasoning test based on grammatical transformation. Psychon Sci 10, 341-2.

49) Rosekind MR, Smith RM, Miller DL, Co EL, Gregory KB,
Webbon LL, Gander PH, Lebacqz JV (1995) Alertness management: strategic naps in operational settings. J Sleep Res 4, 62-6.

50) Chee MW, Choo WC (2004) Functional imaging of working memory after $24 \mathrm{hr}$ of total sleep deprivation. J Neurosci 24, 4560-7.

51) Hirshkowitz M, Moore CA, Hamilton III CR, Rando KC, Karacan I (1992) Polysomnography of adults ans elderly: sleep architecture, respiration, and leg movement. J Clin Neurophysiol 9, 56-62.

52) Sasaki T, Matsumoto $S$ (2001) A preliminary attempt on the evaluation of a nocturnal nap viewed from the SWS-SREM balance and progressive stage changes during daytime sleep. J Sci Labour 77, 131-46 (in Japanese with English abstract).

53) Dinges DF, Orne MT, Whitehouse WG, Orne EC (1987) Temporal placement of a nap for alertness: contributions of circadian phase and prior wakefulness. Sleep 10, 313-29.

54) Tassi P, Muzet A (2000) Sleep inertia. Sleep Med Rev 4, 341-53.

55) Hayashi M, Masuda A, Hori T (2003) The alerting effects of caffeine, bright light and face washing after a short daytime nap. Clin Neurophysiol 114, 2268-78.

56) Stickgold R, Whidbee D, Schirmer B, Patel V, Hobson JA (2000) Visual discrimination task improvement: a multi-step process occurring during sleep. J Cogn Neurosci 12, 24654. 